\title{
Active Focusing of Light in Plasmonic Lens via Kerr Effect
}

\author{
Hadiseh Nasari* and Mohammad Sadegh Abrishamian \\ Department of Electrical and Computer Engineering, K. N. Toosi University of Technology, \\ Tehran 16314, Iran
}

(Received May 3, 2012 : revised June 14, 2012 : accepted June 26, 2012)

\begin{abstract}
We numerically demonstrate the performance of a plasmonic lens composed of an array of nanoslits perforated on thin metallic film with slanted cuts on the output surface. Embedding Kerr nonlinear material in nanoslits is employed to modulate the output beam. A two dimensional nonlinear-dispersive finitedifference time-domain (2D N-D-FDTD) method is utilized. The performance parameters of the proposed lens such as focal length, full-width half-maximum, depth of focus and the efficiency of focusing are investigated. The structure is illuminated by a TM-polarized plane wave and a Gaussian beam. The effect of the beam waist of the Gaussian beam and the incident light intensity on the focusing effect is explored. An exact formula is proposed to derive electric field $\boldsymbol{E}$ from electric flux density $\boldsymbol{D}$ in a Kerr-Dispersive medium. Surface plasmon (SPs) modes and Fabry-Perot (F-P) resonances are used to explain the physical origin of the light focusing phenomenon. Focused ion beam milling can be implemented to fabricate the proposed lens. It can find valuable potential applications in integrated optics and for tuning purposes.

Keywords: Plasmonics, MIM waveguide, N-D-FDTD

OCIS codes : (240.6680) Surface plasmons; (190.0190) Nonlinear optics; (250.5300) Photonic integrated circuits; (230.1150) All-optical devices; (050.2230) Fabry-Perot
\end{abstract}

\section{INTRODUCTION}

While on-chip information processing can efficiently speed up by employing photonic integrated circuits (PICs), there was a significant challenge to realize them by a density comparable to the electronic counterparts due to the presence of the optical diffraction limit. Surface plasmon-based photonics or plasmonics, a recently emerged device technology, can propose a solution to this dilemma, because plasmonics has both the large data carrying capacity of photonics and the miniaturization of electronics [1-3]. Although surface plasmon polaritons (SPPs), surface electromagnetic waves coupled to collective oscillations of free electrons in a metal [4], were first theoretically proposed in 1957 [5], the significant growth and interest in plasmonics is formed since Ebbesen first reported the extraordinary optical transmission through a 2D metallic hole array in 1998 [6]. Nano fabrication techniques, such as electron-beam lithography and focused ion beam milling can be implemented to pattern metallic structures at the nanoscale and make it possible to study the interaction of these nanostructures with light $[7,8]$.

The strong localization of SPPs at the metal-dielectric interfaces has attracted tremendous interests of researchers to SPP-based subwavelength guiding components [9] such as arrayed nanoparticles [10], V-grooves [11], wedges [12], nanowires [13], dielectric-loaded metal films [14] and plasmonic slot waveguides [15]. Among them, Metal-InsulatorMetal (MIM) waveguides are promising in design of a variety of nanoscale plasmonic devices because of possessing high group velocity over a wide frequency range from DC to visible, strong confinement of light waves and also simple fabrication and integration into optical circuits $[16,17]$. Although Insulator-Metal-Insulator (IMI) waveguides have the advantage of less loss for the longer propagation distance, but their light confinement capability into subwavelength scales is poor [18]. Numerous functional plasmonic MIM structures like directional couplers [19], filters [20], Y-shaped combiners [21], sensors [22], U-shaped waveguides [23], Bragg reflectors [24], etc, have been numerically and/or experimentally investigated.

Refractive lenses are one of the most ubiquitous optical components with applications ranging from imaging to concentrating light, but their light confinement capability deteriorates as their size approaches the wavelength of

\footnotetext{
*Corresponding author: Hadiseh_Nasari@ee.kntu.ac.ir

Color versions of one or more of the figures in this paper are available online.
} 
light due to the diffraction limit. Surface-plasmon based lenses or plasmonic lenses as an alternative to the ordinary refractive lenses are capable of super focusing beyond the diffraction limit and have great applications in optical data storage, nanofabrication, single molecular biosensing, circular polarizer analyzer and etc [7, 25-27]. Single subwavelength slit surrounded by surface corrugation [28] or by chirped dielectric surface gratings [29], chirped circular slits corrugated on metallic film [30], quasiperiodic array of nanoholes [31] and nanometric cross-shaped aperture arrays [32] in a metal screen are some of the reported design principles to implement the focusing capability of plasmonic lenses. Nanoslits perforated on thin metallic films are recently employed to manipulate the phase front profile [33-35]. The phase shift experienced by light passing through nanoslits is sensitive to its width and depth and also the refractive index of incorporated material. The required phase front profile for focusing action can be achieved by appropriate adjustment of the properties of each slit.

Active control of plasmons which is one of the greatest challenges in recent years is needed to achieve tunable plasmonic devices, besides that, more specifically; the ability to control light with light is required in all-optical signal processing in PICs and optical computing. This can be realized by employing active materials whose properties can be altered by some form of stimulation $[3,36]$. Switches and modulators on the basis of the electro-optic effect $[37,38]$, the magneto-optic effect [39], the thermo-optic effect [40], the plasma dispersion effect [41], the plasmonic excitation of quantum dots [42] and the Kerr nonlinearity [43] are investigated. In the plasmonic lenses formed by nanoslits perforated on thin films, modulating the output beam can be accomplished by incorporating nanoslits with a type of active material like Kerr nonlinear material [44] or anisotropic nematic liquid crystal [45].

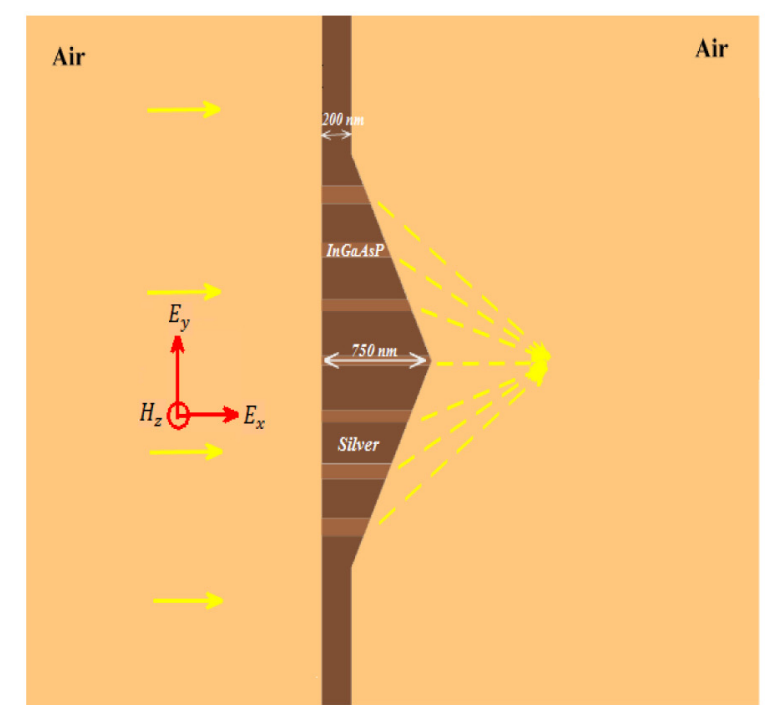

(a)
In this paper we numerically explore the focusing effect of a plasmonic lens formed by nanoslits perforated on a thin metallic film with slanted cuts on the output surface and incorporated by Kerr nonlinear material. The performance parameters of the proposed lens including focal length (FL), depth of focus (DOF) defined at the half maximum intensity, full-width half-maximum (FWHM) and the efficiency of focusing are investigated. The lens is illuminated by a Gaussian beam and plane wave and the possibility of tuning the focal length by the intensity of incident light and the beam waist of the Gaussian beam is investigated.

This paper is organized as follows; In Section 2 the structure of proposed lens and the analysis method are presented. Section 3 elucidates results of the detailed simulations of the lens and the conclusion will be in Section 4 .

\section{DEVICE STRUCTURE AND ANALYSIS METHOD}

The simulated structure is displayed in Fig. 1(a). The slit widths in consequence from top to bottom are 120 , $100,80,60,80,100$ and $120 \mathrm{~nm}$ with $400 \mathrm{~nm}$ center to center spacing between any two adjacent slits. The underlying physics involved in design of our proposed lens can be discussed as follows. A narrow slit surrounded by metallic walls is the basic building block of the lens. If the space between every two adjacent slits is much more than the metal's skin depth at operating wavelength, these slits can be assumed as isolated MIMs besides that the slits widths in our structure are much smaller than the operating wavelength, so just the propagation of fundamental SPP mode in MIM waveguide is considered. The complex propagation constant $\beta$ of the transverse magnetic (TM) mode in this MIM waveguide is determined by the following

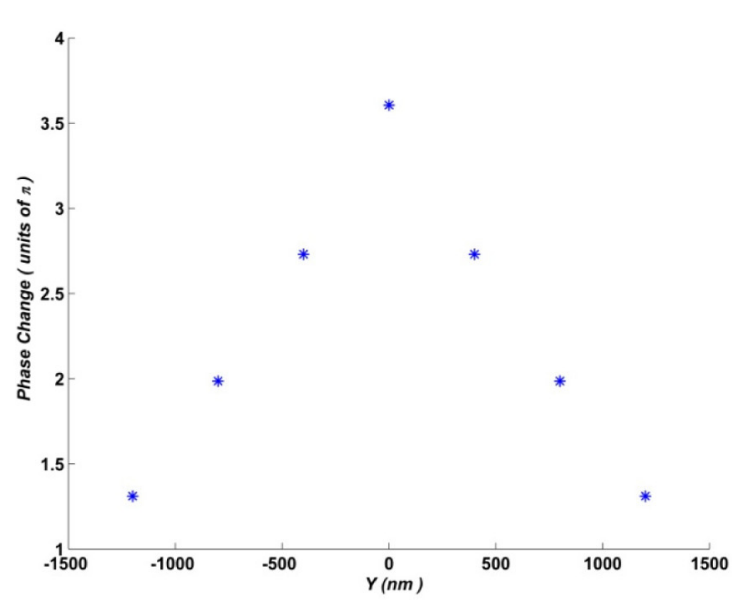

FIG. 1. (a) Schematic and (b) phase front profile of the proposed plasmonic lens. 
dispersion relation [15]:

$$
\tanh \left(\frac{k_{d} w}{2}\right)=-\frac{\varepsilon_{d} k_{m}}{\varepsilon_{m} k_{d}}
$$

Where $k_{d}=\sqrt{\beta^{2}-k_{0}^{2} \varepsilon_{d}}$ and $k_{m}=\sqrt{\beta^{2}-k_{0}^{2} \varepsilon_{m}}$ are the propagation constants of the dielectric and metal respectively. $k_{0}$ $=2 \pi / \lambda_{0}, \varepsilon_{m}, \mathcal{E}_{d}$ and $w$ are wave number in free space, the dielectric constants of metal cladding and dielectric core and the width of the MIM waveguide, respectively. The phase delay introduced by a slit is calculated from [34]:

$$
\Delta \phi=\Delta \phi_{1}+\Delta \phi_{2}+\beta d-\theta
$$

Where $\Delta \phi_{1}$ and $\Delta \phi_{2}$ are accompanied phase changes at the entrance and exit interfaces and cancel each other if the medium on the illuminated and unilluminated sides of the lens be the same, $d$ is the slit depth, $\theta$ originates from multiple reflections of light between the entrance and exit surfaces. But the phase delay is dominated by the $R e(\beta d)$ [34]. So if the slits be the same in width and incorporated material, the phase delay will be proportional to slit depth, on the other hand narrower slits bring more phase delays, in the result of more residence of mode in metal cladding which causes the light to travel slower [7]. In our proposed lens the slit depth and width increases and decreases respectively toward the center of the structure and hence creates the required phase front profile for light focusing which is shown in Fig. 1(b).

The performance of the proposed lens has been simulated by a two-dimensional nonlinear-dispersive finite-difference time-domain (2D N-D-FDTD) numerical method, assuming the slit lengths (in $\mathrm{z}$ direction) to be infinite. This assumption is acceptable for slit lengths larger than $15 \mu \mathrm{m}$ [46] and by using the fact that the error in $2 \mathrm{D}$ simulations in comparison with 3D simulations will be negligible (Less than 10 percent). Obviously more accurate results by investigating the effect of finite slit length can be obtained by 3D N-D-FDTD but at a cost of large amounts of computer memory and increased computing time. The outer boundary of the computation lattice is terminated to the convolutional perfectly matched layer (CPML) to dissipate outgoing waves [47]. The second-order Lorentz dispersion model is employed to characterize the frequency-dependent relative permittivity of silver, when time dependency is taken $\exp (j \omega t)$ [48]:

$$
\varepsilon_{R}(\omega)=\varepsilon_{r}-j \mathcal{E}_{i}=\frac{\varepsilon_{\infty}}{\varepsilon_{0}}+\frac{\chi_{0} \omega_{0}^{2}}{\omega_{0}^{2}-\omega^{2}+j \Gamma \omega}
$$

Where $\omega_{0}$ and $\Gamma$ are resonant frequency and damping coefficient. $\varepsilon_{\infty}=\varepsilon_{0}\left(1+\chi_{\infty}\right)$ determines the response of the medium for frequencies far above resonant frequency and $\chi_{0}$ is the DC response of the polarization to the electric field. Knowing $\mathcal{E}_{R}$ at operating frequency which can be easily obtained from hand books like [49] and setting reasonable values for $\varepsilon_{\infty}$ and $\chi_{0}$ like $\chi_{0}=10$ and $\varepsilon_{\infty}=2.89 \varepsilon_{0}$, we can calculate $\omega_{0}$ and $\Gamma$ from [48]:

$$
\begin{aligned}
& \omega_{0}=\frac{\omega}{\left\{1-\chi_{0}\left(\varepsilon_{r}-\varepsilon_{\infty} / \varepsilon_{0}\right) /\left[\left(\varepsilon_{r}-\varepsilon_{\infty} / \varepsilon_{0}\right)^{2}+\varepsilon_{i}^{2}\right]\right\}^{1 / 2}} \\
& \Gamma=\frac{\omega \chi_{0} \varepsilon_{i}}{\left(\varepsilon_{r}-\varepsilon_{\infty} / \varepsilon_{0}\right)^{2}+\varepsilon_{i}^{2}-\chi_{0}\left(\varepsilon_{r}-\varepsilon_{\infty} / \varepsilon_{0}\right)}
\end{aligned}
$$

A TM-polarized plane wave of $850 \mathrm{~nm}$ wavelength consisting of $E_{x}, E_{y}$, and $H_{z}$ field components illuminates the structure. The relative permittivity of silver at this wavelength is $\varepsilon_{m}=-33.22-j 1.17$. The grid sizes are chosen $\Delta x$ $=\Delta y=5 \mathrm{~nm}$ and the time step is set $\Delta \mathrm{t}=0.85 /$ $\left(c \sqrt{(\triangle x)^{-2}+(\Delta y)^{-2}}\right)$ achieved by Courant stability condition, where $c$ is the speed of light in free space.

The nanoslits are filled with Kerr nonlinear material. For a Kerr nonlinear medium the dielectric constant $\varepsilon_{d}$ depends on the intensity of incident light by [50]:

$$
\left.\varepsilon_{d}=\varepsilon_{l}+\chi^{(3)}\right)|E|^{2}
$$

The linear dielectric constant $\mathcal{E}_{l}$ is set as 2.25 . The thirdorder nonlinear susceptibility $\chi^{(3)}=1.4 \times 10^{-10}$ esu is chosen as a typical value of nonlinear optical materials such as InGaAsP [50].

To simulate the performance of proposed lens, at first step, The electric flux density $\mathbf{D}$ is obtained by solution to Maxwell's equations; $\nabla \times \boldsymbol{E}=-\mu \partial \boldsymbol{H} / \partial t$, and $\nabla \times \boldsymbol{H}=\partial \boldsymbol{D} / \partial t$ where $\boldsymbol{E}$ and $\boldsymbol{H}$ are the electric and magnetic fields, respectively and $\mu$ is the magnetic permeability, according to Yee algorithm. The electric field can be derived from following equation [47]:

$$
\boldsymbol{D}=\varepsilon_{0} \varepsilon_{\infty} \boldsymbol{E}+\boldsymbol{P}^{L}+\boldsymbol{P}^{N L}
$$

Where $\varepsilon_{0}$ is the free space permittivity, $\boldsymbol{P}^{L}$ and $\boldsymbol{P}^{N L}$ are linear and nonlinear polarization vectors associated with the Lorentz model of silver and Kerr nonlinearity, respectively [47].

$$
\boldsymbol{P}_{\text {Lorentz }}^{n+1}=A \boldsymbol{P}_{\text {Lorentz }}^{n}+B \boldsymbol{P}_{\text {Lorentz }}^{n-1}+C \boldsymbol{E}^{n}
$$

$$
\boldsymbol{P}_{\text {Kerr }}=\varepsilon_{0} \chi_{0}^{(3)} \boldsymbol{E}^{3}
$$

Where $A=\left(2-\omega^{2}{ }_{0} \Delta t^{2}\right) /((\Gamma / 2) \Delta t+1), B=(\Gamma \Delta t-2) /(\Gamma \Delta t+2)$, $C=\left(\varepsilon_{0} \chi_{0} \omega_{0}^{2} \Delta t^{2}\right) /((\Gamma / 2) \Delta t+1)$ and $\Delta t$ is the time step. By substituting Eq. (8) and Eq. (9) in Eq. (7), the electric field can be obtained by considering two methods, by using Newton iteration [47]:

$$
\boldsymbol{E}^{\ltimes m+1>}=\left(\boldsymbol{D}^{n+1}-\boldsymbol{P}_{\text {Lorentz }}^{n+1}\right) /\left(\varepsilon_{0} \varepsilon_{\infty}+\varepsilon_{0} \chi^{(3)}\left(\boldsymbol{E}^{<m>}\right)^{2}\right)
$$


Where $m=0,1,2, \cdots, \boldsymbol{E}^{r m>}$ is the approximation of $\boldsymbol{E}^{n+1}$ at the mth iteration and $\boldsymbol{E}^{<0>}=\boldsymbol{E}^{n}$. This iteration process continues until sufficient accuracy is obtained. Another way is by considering Eq. (7) as a cubic equation:

$$
a\left(\boldsymbol{E}^{n+1}\right)^{3}+b\left(\boldsymbol{E}^{n+1}\right)+c=0
$$

Where $a=\varepsilon_{0} \chi^{(3)}, b=\varepsilon_{0} \mathcal{E}_{\infty}$, and $c=\boldsymbol{P}_{\text {Lorent } z^{-}}^{n+1} \boldsymbol{D}^{n+1}$ that has a solution for $\boldsymbol{E}^{n+1}$ :

$$
\begin{aligned}
E^{n+1}= & -\frac{.87358 b}{\left(-9 a^{2} c+1.73205 \sqrt{4 a^{3} b^{3}+27 a^{4} c^{2}}\right)^{1 / 3}}+ \\
& \frac{.381571\left(-9 a^{2} c+1.73205 \sqrt{4 a^{3} b^{3}+27 a^{4} c^{2}}\right)^{1 / 3}}{a}
\end{aligned}
$$

This result is used in following simulations and provides more accuracy than the first method for few iterations.

\section{SIMULATION RESULTS AND DISCUSSION}

As mentioned previously, change in the dielectric constant of incorporated material in nanoslits can be implemented to modulate the output beam. For different values of $\mathcal{E}_{d}$ the dispersion equation is solved for $\beta$ by using Mathematica. The phase delay of transmitted light through nanoslits in the situation of illuminating the structure by plane wave is calculated according to Eq. (2) and the results are presented in Fig. 2(a). The results of change in the propagation length defined as $L=(2 \operatorname{Im}(\beta))^{-1}$ [51] by varying $\mathcal{E}_{d}$ are illustrated in Fig. 2(b).

The nanoslits in our structure are filled with Kerr nonlinear material. By increasing the intensity of incident light, in the situation that Fabry-Perot (F-P) resonances occur in the slits in the result of impedance mismatch at the entrance and exit surfaces of each slit, the dielectric constant of incorporated nonlinear material increase. By increasing $\mathcal{E}_{d}$, the relative phase difference and hence the phase front profile changes so tuning the focal length by incident light intensity becomes possible. When the incident light intensity is $13.2 \mathrm{MW} / \mathrm{cm}^{2}$, all of the slits are out of F-P resonances and the calculated electric field intensity on the $\mathrm{x}$-axis of the lens and on the focal plane which represent the FL, DOF and FWHM respectively are shown in Fig. 3(a), (b).

The results depicted in Fig. 4(a) illustrate the movement of focal point toward lens by increasing the intensity of incident light, which is in the result of formation F-P resonances in three central slits, while the outer slits remain out of F-P resonances. Figure 4(b) represents the dependence of the dielectric constant of incorporated Kerr nonlinear material in nanoslits located at $\mathrm{y}=0$ and $400 \mathrm{~nm}$ to the incident light intensity.

Table 1 lists the focusing properties of the lens for different values of incident light intensity. FWHM is approximately unchanged and remains equal to $590 \mathrm{~nm}$ for all four cases but the DOF parameter which determines the working distance for beaming application, decreases.

Certainly, rather than the absorption of a portion of light in the structure, there is reflection of light from the surface of the lens in the illuminated side, so the proposed lens cannot transmit $100 \%$ of the incident light. Also, it should be pointed out that in these kinds of lenses which are formed on the basis of nanoslits perforated on thin metallic films, because of the low decoupling rate of surface plasmon modes to propagating electromagnetic wave modes, most of the energy remains at the output surface of the lens and hence the efficiency of energy passing through the lens will be much more than the efficiency of focusing [25]. The efficiency of focusing which is obtained by dividing

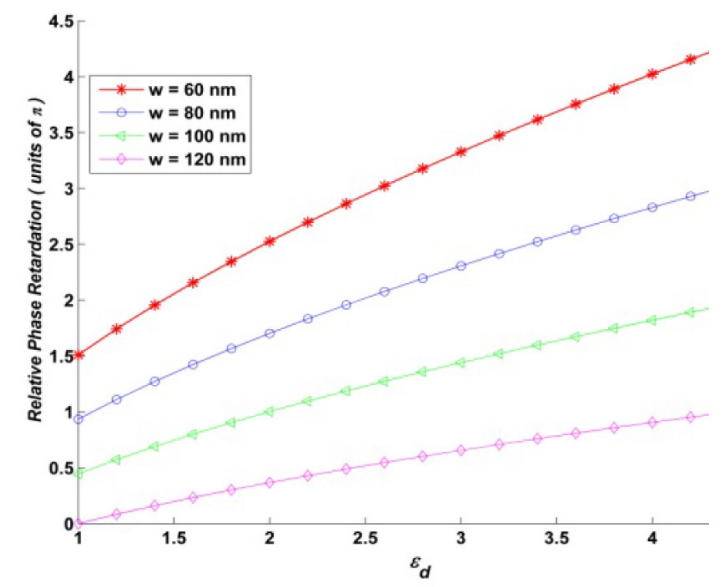

(a)

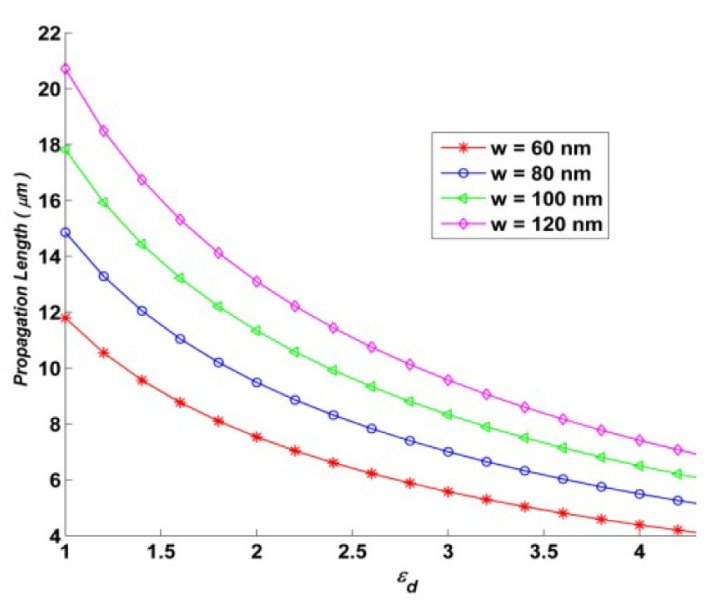

(b)

FIG. 2. (a) Relative phase retardation caused by varying and (b) propagation length of SPPs as a function of the dielectric constant of incorporated material in nanoslits. 


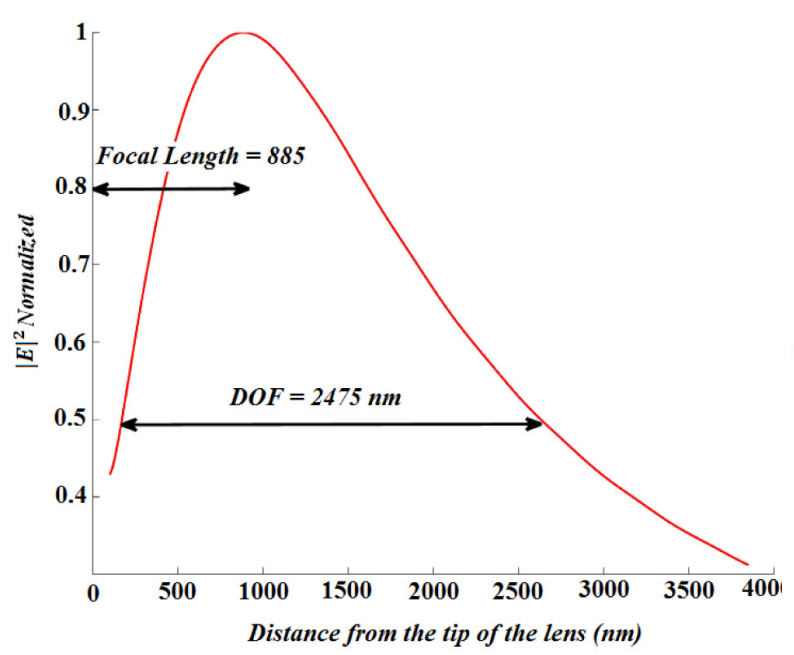

(a)

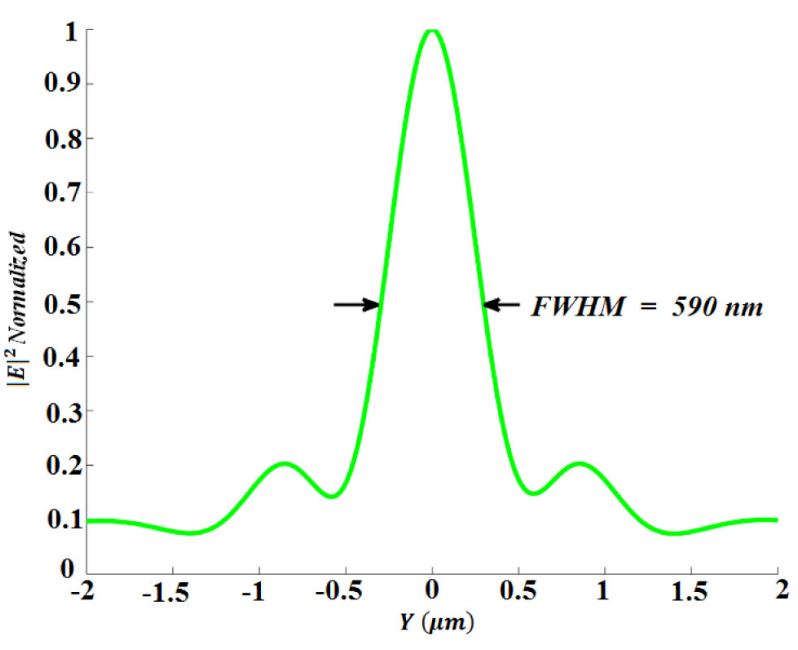

(b)

FIG. 3. Calculated normalized electric field intensity on the (a) x-axis (b) focal plane of the lens for the $13.2 \mathrm{MW} / \mathrm{cm}^{2}$ intensity of incident plane wave.

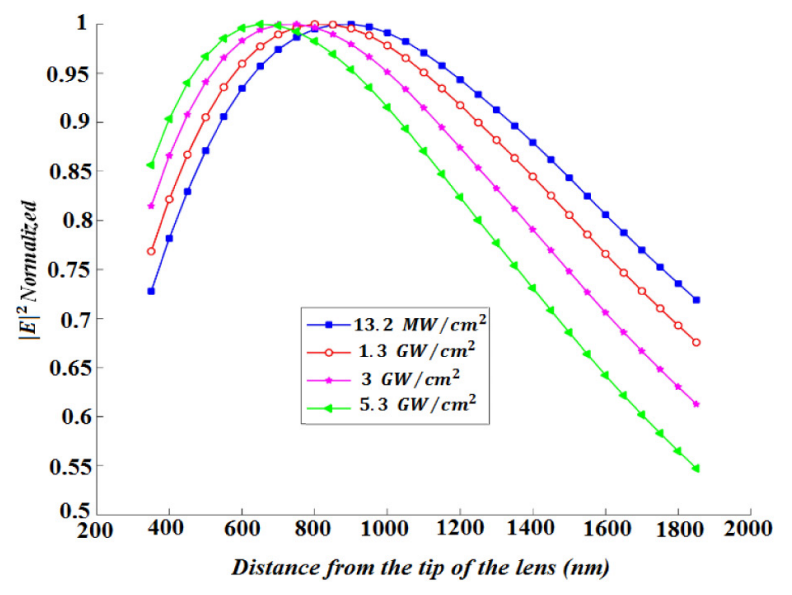

(a)

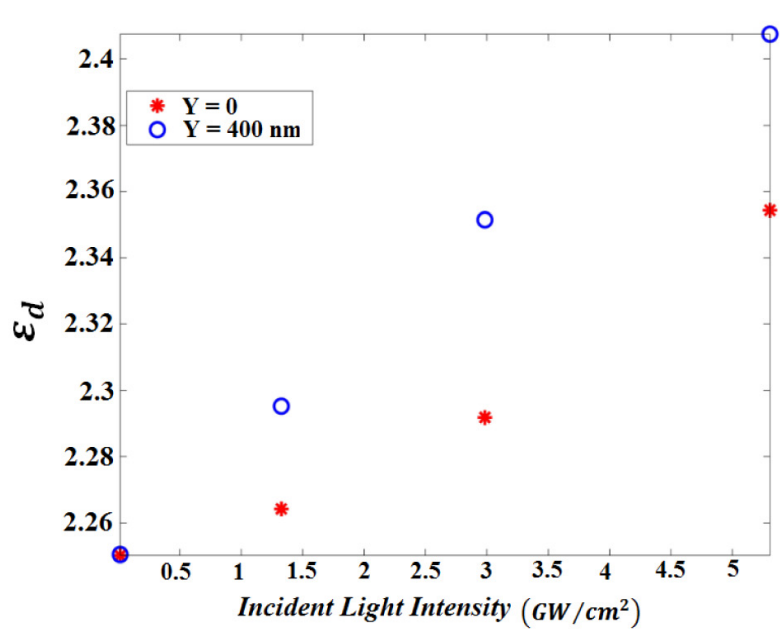

(b)

FIG. 4. (a) Decrease in focal length by increasing the intensity of incident plane wave and (b) the dependence of the dielectric constant of incorporated Kerr nonlinear material in nanoslits located at $\mathrm{y}=0$ and $400 \mathrm{~nm}$ to the incident light intensity.

TABLE 1. Performance parameters of the proposed lens for different values of incident plane wave intensity

\begin{tabular}{c|c|c|c|c}
\hline \hline Intensity of Incident Light & $13.2 \mathrm{MW} / \mathrm{cm}^{2}$ & $1.3 \mathrm{GW} / \mathrm{cm}^{2}$ & $3 \mathrm{GW} / \mathrm{cm}^{2}$ & $5.3 \mathrm{GW} / \mathrm{cm}^{2}$ \\
\hline DOF (nm) & 2475 & 2310 & 2090 & 2025 \\
\hline Efficiency of Focusing (\%) & 42.4 & 46 & 51.1 & 53.5 \\
\hline FL (nm) & 885 & 820 & 735 & 660 \\
\hline
\end{tabular}

the intensity of focal point to the incident intensity increases by increasing the incident light intensity; attributed to the formation of F-P resonances in central slits and the results are also presented in Table 1 .

In comparison with the situation of illuminating the lens by a plane wave, when the lens is illuminated by a Gaussian beam, the central slits provide more phase retardation and hence the focal length decreases. Also the amount of decrease in focal length depends on the beam waist of the Gaussian beam which is shown in Fig. 5.

The performance parameters of the lens for the cases of illuminating the structure by plane wave, Gaussian beam with beam waist of $2 \mu \mathrm{m}$ and $1.5 \mu \mathrm{m}$ are listed in Table 2 .

We are limited in how much we can increase the incident 


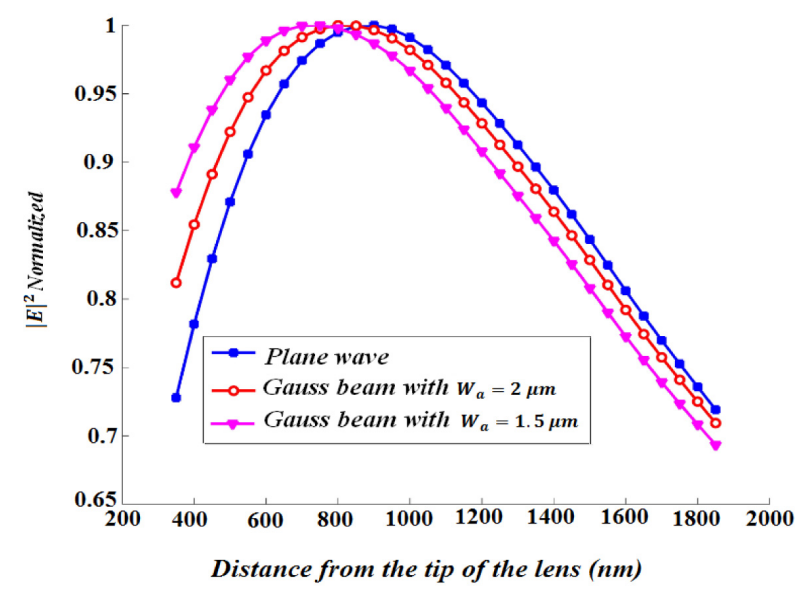

FIG. 5. Calculated normalized electric field intensity on the $\mathrm{x}$-axis of the lens for the cases of illuminating the lens with plane wave, Gaussian beam with $w_{a}=2 \mu \mathrm{m}$ and $w_{a}=1.5 \mu \mathrm{m}$.

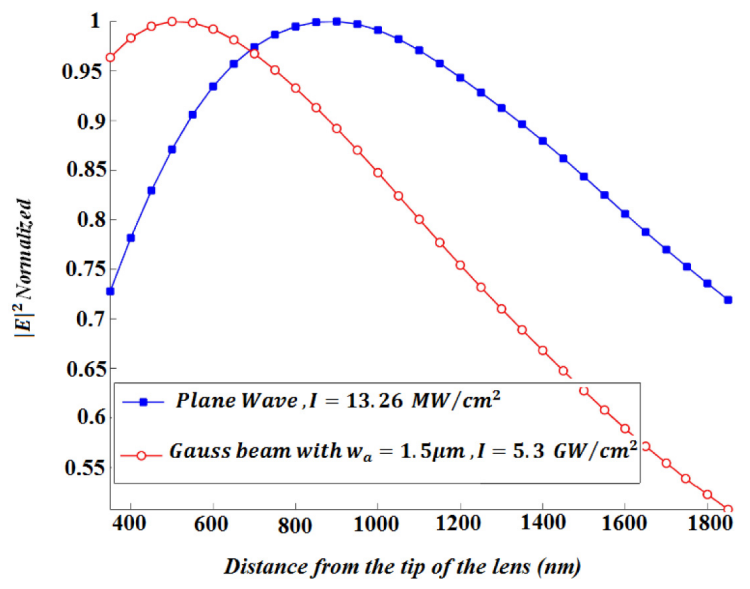

FIG. $6.370 \mathrm{~nm}$ shift in focal point for the cases of illuminating the lens with $13.2 \mathrm{MW} / \mathrm{cm}^{2}$ plane wave and $5.3 \mathrm{GW} / \mathrm{cm}^{2}$ Gaussian beam with $w_{a}=1.5 \mu \mathrm{m}$.

TABLE 2. Performance parameters of the proposed lens for the cases of illuminating the lens with plane wave, Gaussian beam with $w_{a}=2 \mu \mathrm{m}$ and $w_{a}=1.5 \mu \mathrm{m}$

\begin{tabular}{c|c|c|c}
\hline \hline Incident Light & Plane Wave & Gaussian Beam, $w_{a}=2 \mu \mathrm{m}$ & Gaussian Beam, $w_{a}=1.5 \mu \mathrm{m}$ \\
\hline DOF (nm) & 2475 & 2530 & 2500 \\
\hline FWHM (nm) & 590 & 595 & 605 \\
\hline FL (nm) & 885 & 825 & 735 \\
\hline Efficiency of Focusing (\%) & 42.4 & 31 & 25 \\
\hline
\end{tabular}

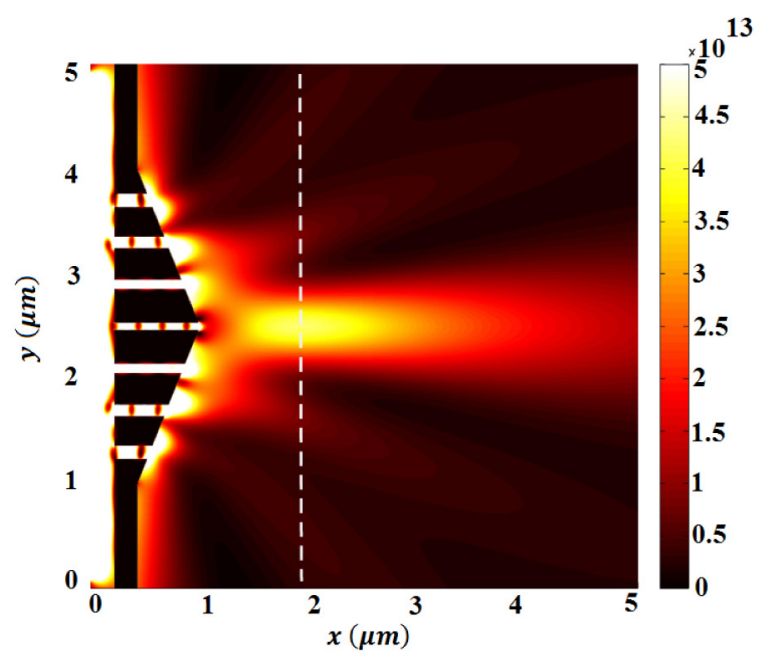

(a)

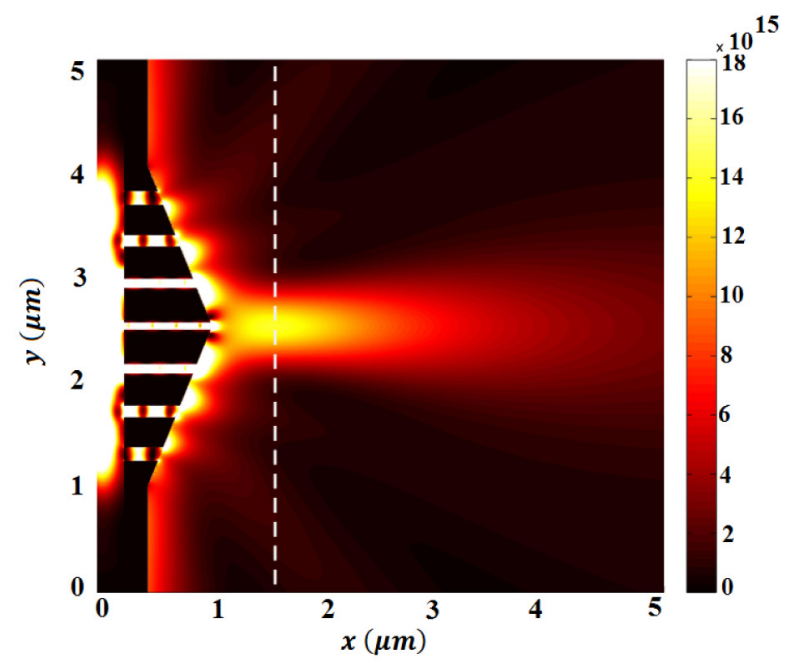

(b)

FIG. 7. Time average electric field intensity distribution for illuming the lens by (a) $13.2 \mathrm{MW} / \mathrm{cm}^{2}$ plane wave and (b) $5.3 \mathrm{MW} / \mathrm{cm}^{2}$ Gaussian beam with $w_{a}=1.5 \mu \mathrm{m}$.

light intensity to reduce the focal length, but we can increase the maximum change in focal length by employing a Gaussian beam. Our simulations reveal that when the structure is illuminated by $5.3 \mathrm{GW} / \mathrm{cm}^{2}$ Gaussian beam with $1.5 \mu \mathrm{m}$ beam waist and plane wave the focal length will be 515 and 660 $\mathrm{nm}$ respectively, so if illuminating the structure by 13.2
$\mathrm{MW} / \mathrm{cm}^{2}$ plane wave be considered as the initial situation, the maximum change in focal length increases $64 \%$ in the case of employing a Gaussian beam instead of a plane wave as is shown in Fig. 6.

Time-average electric field intensity distribution $|E|^{2}$ for two cases depicted in Fig. 6, are presented in Fig. 7. 
The maximum intensity of incident light in our simulations is $5.3 \mathrm{GW} / \mathrm{cm}^{2}$ which is below the threshold that can result in material damage, but temperature increase as the result of optical absorption, should be considered. Electron thermal dynamics induced by the interaction of a high intensity light with a metal can be precisely described using the Two-Temperature Model. Two temperatures refer to that of the electrons $\left(T_{e}\right)$ and the lattice $\left(T_{l}\right)$, and are functions of time and depth into the sample. The lattice thermalizes with the electron but because of the large value of lattice heat capacity, $\sim 2.4 \mathrm{~J} / \mathrm{cm}^{3} / \mathrm{C}^{\circ}$ for silver, changes of hundreds of degree to $T_{e}$ lead to changes of only tens of degrees to $T_{l}$. In experimental cases, by heat transfer to the substrate and cooling the lattice, this temperature rise will be lower $[52,53]$.

\section{CONCLUSION}

In conclusion, the performance of a plasmonic lens composed of an array of nanoslits introduced in thin metallic film with slanted cuts on the output surface and filled with Kerr nonlinear material has been explored. The performance parameters of the lens for the cases of illuminating by plane wave and Gaussian beam with different intensity and beam waist have been investigated. An exact formula for obtaining electric field $\boldsymbol{E}$ from electric flux density $\boldsymbol{D}$ in Kerr-dispersive medium which provide more accuracy than conventional ittirative method has been proposed. The maximum focusing efficiency of $53.5 \%$ has been achieved for the situation that the structure is illuminated by a plane wave with the intensity of $5.3 \mathrm{GW} / \mathrm{cm}^{2}$. The focal length can be tuned on the x-axis of the lens by amount of 370 $\mathrm{nm}$. According to our knowledge there are not any structural damages for the values of incident light intensity up to 5.3 $\mathrm{GW} / \mathrm{cm}^{2}$. Our proposed plasmonic lens offers great applications at many regions like near field imaging, sensing, subwavelength optics and etc.

\section{REFERENCES}

1. Y. Song, J. Wang, M. Yan, and M. Qiu, "Efficient coupling between dielectric and hybrid plasmonic waveguides by multimode interference power splitter," J. Opt. 13, 75002 (2011).

2. R. Zia, J. A. Schuller, A. Chandran, and M. L. Brongersma, "Plasmonics: the next chip-scale technology," Mater. Today 9, 20-27 (2006).

3. E. Ozbay, "Plasmonics: merging photonics and electronics at nanoscale dimentions," Science 311, 189-193 (2006).

4. T. Tanemura, K. C. Balram, D. S. L. Gagnon, P. Wahl, J. S. White, M. L. Brongersma, and D. A. B. Miller, "Multiplewavelength focusing of surface plasmons with a nonperiodic nanoslit coupler," Nano Lett. 11, 2693-2698 (2011).

5. R. H. Ritchie, "Plasma losses by fast electrons in thin films," Phys. Rev. 106, 874-881 (1957).

6. T. W. Ebbesen, H. J. Lezec, H. F. Ghaemi, T. Thio, and P. A. Wolff, "Extra ordinary optical transmission through subwavelength hole arrays," Nature 391, 667-669 (1997).

7. L. Verslegers, P. B. Catrysse, Z. Yu, J. S. White, E. S. Barnard, M. L. Brongersma, and S. Fan, "Planer lenses based on nanoscale slit arrays in a metallic film," Nano Lett. 9, 235-238 (2009).

8. A. Normatov, P. Ginzburg, N. Berkovitch, G. M. Lerman, A. Yanai, U. Levy, and M. Orenstein, "Efficient coupling and field enhancement for the nano-scale: plasmonic needle," Opt. Express 18, 14079-14086 (2010).

9. W. L. Barnes, A. Dereux, and T. W. Ebbesen, "Surface plasmon subwavelength optics," Nature 424, 824-830 (2003).

10. S. A. Maier, P. G. Kik, H. A. Atwater, S. Meltzer, E. Harel, B. E. Koel, and A. A. G. Requicha, "Local detection of electromagnetic energy transport below the diffraction limit in metal nanoparticle plasmon waveguides," Nature Mat. 2, 229-232 (2003).

11. S. I. Bozhevolnyi, V. S. Volkov, E. Devaux, and T. W. Ebbesen, "Channel plasmon-polariton guiding by subwavelength metal grooves," Phys. Rev. Lett. 95, 046802 (2005).

12. A. Boltasseva, V. S. Volkov, R. B. Nielsen, E. Moreno, S. G. Rodrigo, and S. I. Bozhevolnyi, "Triangular metal wedges for subwavelength plasmon-polariton guiding at telecom wavelengths," Opt. Express 16, 5252-5260 (2008).

13. J. C. Weeber, A. Dereux, C. Girard, J. Krenn, and J. P. Goudonnet, "Plasmon polaritons of metallic nanowires for controlling submicron propagation of light, " Phys. Rev. B 60, 9061-9068 (1999).

14. T. Holmgaard, S. I. Bozhevolnyi, L. Markey, and A. Dereux, "Efficient excitation of dielectric-loaded surface plasmonpolariton waveguide modes at telecommunication wavelength," Phys. Rev. B 78, 165431 (2008).

15. J. A. Dionne, L. A. Sweatlock, H. A. Atwater, and A. Polman, "Plasmon slot waveguides: toward chip-scale propagation with subwavlength-scale localization," Phys. Rev. B 73, 035407 (2006).

16. J. H. Zhu, Q. J. Wang, P. Shum, and X. G. Huang, "A nanoplasmonic high-passwavelength filter based on a metalinsulator-metal circuitous waveguide," IEEE Trans. Nano Tech. 10, 1357-1361 (2011).

17. C. Min and G. Veronis, "Absorption switches in metaldielectric-metal plasmonic waveguides," Opt. Express 17, 10757-10766 (2009).

18. H. Lu, X. Liu, D. Mao, L. Wang, and Y. Gong, "Tunable band-pass plasmonic waveguide filters with nanodisk resonators," Opt. Express 18, 17922-17927 (2010).

19. P. Chen, R. Liang, Q. Huang, Z. Yu, and X. Xu, "Plasmonic filters and directional couplers based on wide metal-insulatormetal structure," Opt. Express 19, $7633-7639$ (2011).

20. A. Setayesh, S. R. Mirnaziri, and M. S. Abrishamian, "Numerical investigation of tunable band-pass/band-stop plasmonic filters with hollow core circular ring resonator," J. Opt. Soc. Korea 15, 82-89 (2011).

21. H. Gao, H. Shi, C. Wang, C. Du, X. Luo, Q. Deng, Y. Lv, X. Lin, and H. Yao, "Surface plasmon polariton propagation and combination in Y-shaped metallic channels," Opt. Express 13, 10795-10800 (2005).

22. K. M. Byun, "Development of nanostructured plasmonic 
substrates for enhanced optical biosensing," J. Opt. Soc. Korea 14, 65-76 (2010).

23. T. W. Lee and S. K. Gray, "Subwavelength light bending by metal slit structures," Opt. Express 13, 9652-9659 (2005).

24. J. Q. Liu, L. L. Wang, M. D. He, W. Q. Huang, D. Wang, B. S. Zou, and S. Wen, "A wide bandgap plasmonic bragg reflactor," Opt. Express 16, $4888-4894$ (2008).

25. Y. Zhao, S. C. S. Lin, A. A. Nawaz, B. Kiraly, Q. Hao, Y. Liu, and T. J. Huang, "Beam bending via plasmonic lenses," Opt. Express 18, 23458-23465 (2010).

26. S. Yang, W. Chen, R. L. Nelson, and Q. Zhan, "Miniature circular polarization analyzer with spiral plasmonic lens," Opt. Lett. 34, 3047-3049 (2009).

27. Y. Yu and H. Zappe, "Effect of lens size on the focusing performance of plasmonic lenses and suggestions for the design," Opt. Express 19, 9434-44 (2011).

28. F. J. G. Vidal, L. M. Moreno, H. J. Lezec, and T. W. Ebbesen, "Focusing light with a single subwavelngth aperture flanked by surface corrugations," Appl. Phys. Lett. 83, 4500-4502 (2003).

29. S. Kim, Y. Lim, H. Kim, J. Park, and B. Lee, "Optical beam focusing by a sigle subwavelength metal slit surrounded by chirped dielectric surface grating," Appl. Phys. Lett. 92, 013103 (2008).

30. Y. Fu, Y. Liu, X. Zhou, Z. Xu, and F. Fang, "Experimental investigation of superfocusing of plasmonic lens with chirped circular nanoslits," Opt. Express 18, 3438-3443 (2010).

31. F. M. Huang, T. S. Kao, V. A. Fedotov, and Y. Chen, "Nano hole array as a lens," Nano Lett. 8, 2469-2472 (2008).

32. L. Lin, X. M. Goh, L. P. McGuinness, and A. Roberts, "Plasmonic lenses formed by two-dimensional nanometric cross-shaped aperture arrays for Fresnel-region focusing," Nano Lett. 10, 1936-1940 (2010).

33. H. Shi, C. Wang, C. Du, X. Luo, X. Dong, and H. Gao, "Beam manipulating by metallic nano-slits with variant widths," Opt. Express 13, 6815-6820 (2005).

34. T. Xu, C. Wang, C. Du, and X. Luo, "Plasmonic beam deflector," Opt. Express 16, 4753-4759 (2008).

35. Z. Sun and H. K. Kim, "Refractive transmission of light and beam shaping with metallic nano-optic lenses," Appl. Phys. Lett. 85, 642-644 (2004).

36. H. Lu, X. Liu, L. Wang, Y. Gong, and D. Mao, "Ultrafast all-optical switching in nanoplasmonic waveguide with Kerr nonlinear resonator," Opt. Express 19, 2911-2915 (2011).

37. J. H. Zhu, X. G. Huang, and X. Mei, "Plasmonic electrooptical switches operating at telecom wavelengths," Plasmonics 6, 605-612 (2011).

38. M. J. Dicken, L. A. Sweatlock, D. Pacifici, H. J. Lezec, K. Bhattacharya, and H. A. Atwater, "Electrooptic modulation in thin film barium titanate plasmonic interferometers," Nano Lett. 8, 4048-4052 (2008).

39. K. J. Chau, S. E. Irvine, and A. Y. Elezzabi, "A gigahertz surface magneto-plasmon optical modulator," IEEE J. Quantum Electron. 40, 571-579 (2004).

40. T. Nickolajsen, K. Leosson, and S. I. Bozhevonlyi, "In-line extinction modulator based on long- range surface plasmon polaritons," Opt. Commun. 244, 455-459 (2005).

41. Q. Xu, B. Schmidt, S. Pradhan, and M. Lipson, "Mocrometerscale silicon electro-optic modulator," Nature Lett. 435, 325-327 (2005).

42. D. Pacifici, H. J. Lezec, and H. A. Atwateri, "All-optical modulation by plasmonic excitation of CdSe quantum dots," Nature Photon. 1, $402-406$ (2007).

43. J. Tao, Q. J. Wang, and X. G. Huang, "All-optical plasmonic switches based on coupled nano-disk cavity structures containing nonlinear material," Plasmonics 6, 753-759 (2011).

44. C. Min, P. Wang, X. Jiao, Y. Deng, and H. Ming, "Beam manipulating by metallic nano-optic lens containing nonlinear media," Opt. Express 15, 9541-9546 (2007).

45. M. Bahramipanah, S. A. Mirtaheri, and M. S. Abrishamian, "Electrical beam steering with metal-anisotropic-metal structure," Opt. Lett. 37, 527-529 (2012).

46. Y. Pang, C. Genet, and T. W. Ebbesen, "Optical transmission through subwavelength slit apertures in metallic films," Opt. Commun. 280, 10-15 (2007).

47. A. Toflaove and S. C. Hagness, Computational Electrodynamics: The Finite-difference Time-domain Method, 3rd ed. (Artech House, Boston, USA, 2005).

48. J. B. Judkins and R. W. Ziolkowski, "Finit-difference time-domain modeling of nonperfectly conducting metallic thin film grating," J. Opt. Soc. Am. A 12, 1974-1983 (1995).

49. M. J. Weber, Handbook of Optical Materials (CRC Press, Boca Raton, USA, 2003).

50. G. Wang, H. Lu, X. Liu, Y. Gong, and L. Wang, "Optical bistability in metal-insulator-metalic plasmonic waveguide with nanodisk resonator containing Kerr nonlinear medium," Opt. Express 50, 5287-5290 (2011).

51. S. A. Maier, Plasmonics: Fundamentals and Applications (Springer, New York, USA, 2007).

52. M. A. Swillam, N. Rotenberg, and H. M. van Driel, "All-optical ultrafast control of beaming through a single subwavelength aperture in a metal film," Opt. Express 19, 7856-7864 (2011).

53. M. Mansuripur, A. R. Zakharian, A. Lesuffleur, S.-H. Oh, R. J. Jones, N. C. Lindquist, H. Im, A. Kobyakov, and J. V. Moloney, "Plasmonic nano-structures for optical data storage," Proc. SPIE 7505, 75050I (2009). 